\title{
Median codeword shift (MCS) technique for PAPR reduction with low complexity in OFDM system
}

\author{
Mohd Danial Rozaini, Azlina Idris, Darmawaty Mohd Ali, Ezmin Abdullah \\ Faculty of Electrical Engineering, Universiti Teknologi MARA (UiTM), Shah Alam, Malaysia
}

\begin{tabular}{l}
\hline \hline Article Info \\
\hline Article history: \\
Received May 7, 2018 \\
Revised Apr 17, 2019 \\
Accepted Jun 26, 2019 \\
\hline
\end{tabular}

Keywords:

Data permutation

Inter-symbol interference

Median codeword shift

OFDM

PAPR

\begin{abstract}
With the rapid development of today's communication technology, the need for a system capable to improve spectral efficiency, high data rates and at the same time can reduce inter-symbol interference (ISI) is necessary. Orthogonal Frequency Division Multiplexing (OFDM) meet all the requirements needed. However, the high peak to average power ratio (PAPR) has become its major obstacle. This paper is focusing on the development of Median Codeword Shift (MCS), which a new PAPR reduction technique with the capability to reduce the computational complexity of the system. This can be achieved through codeword structure alterization and bit position manipulation by utilizing the circulant shift process. The simulation results revealed that the proposed technique overwhelm conventional OFDM and SCS with $24 \%$ improvement and $0.5 \mathrm{~dB}$ gap from SCS. In fact, the proposed technique possess a lower computational complexity by reducing $16.67 \%$ of the use of IFFT block in the system in contrast with SCS technique.
\end{abstract}

Copyright $\odot 2019$ Institute of Advanced Engineering and Science. All rights reserved.

\section{Corresponding Author:}

Mohd Danial Rozaini,

Faculty of Electrical Engineering, Universiti Teknologi MARA (UiTM), 40450, Shah Alam, Selangor, Malaysia.

Email: danial88phd@gmail.com

\section{INTRODUCTION}

As the time passed, the wireless communication system has rapidly evolved and faced many challenging demand in order to meet the requirement of current technology. Orthogonal Frequency Division Multiplexing (OFDM) is multicarrier modulation technique and is considered as fundamental technology for the wireless communication systems. Due to its unique features such as high data rate, high spectral efficiency, low computational complexity and the immunity to frequency selective fading, OFDM has become an ideal choice for modulation technique in order to meet the requirement for high bit rate transmission system for the past few years. Because of it superiority, OFDM has been adopted in many standard and application such as Wireless Local Area Network (WLAN), Digital Video Broadcasting (DVB), IEEE 802.16 Worldwide Interoperability for Microwave Access (WiMAX) and Long Term Evolution (LTE). However, this multicarrier modulation technique also experienced high peak to average power ratio (PAPR) which can compromise its system performance [1-5]. This is caused by the nature of the modulation in OFDM, where multiple subcarriers or sinusoid are added together to form the signal to be transmitted. These large peaks increase the probability of energy spilling to the adjacent channel, signal distortion in the power amplifier and error rate performance degradation due to the system constraint to a limited peak power of dynamic range in transmitter amplifiers [6,7]. Thus, having a large power amplifier are required. However, by increasing the large power in amplifier not only reduces the power efficiency but also increase the amplifier cost significantly. In addition to the cost, the bad effects that mentions earlier are become the main barrier to the adaption of OFDM system in mobile wireless system. 
Researchers have proposed many techniques to deal with these problems [1-3], so far two major methods have been identified as being potentially important: distortion method and distortion-less method. Clipping and filtering are the two technique that been categorized under distortion method. The fundamental of clipping technique is to limit the peak value of input signal to the predetermined value while filtering is to reduce out of band radiation. Clipping and filtering process need to be repeated because of some peak regrowth of the signal that may exceed threshold value of clipping [1]. These method is quite effective to improve PAPR performance relatively but the major drawback of this technique is it will compromise the bit error rate (BER) performance and will cause the reformation of undesirable spectrum to the adjacent channel [8]. On the other hand, distortion-less technique such as coding [9], partial transmit sequence (PTS) [10, 11], selective mapping (SLM) [12-14] and selective codeword shift (SCS) are capable to reduce the PAPR value and at the same time not affecting the BER performance. However, these techniques are still having limitations in terms of data rate loss, higher computational complexity, larger memory size and requirement of side information for data recovery process at the receiver [7, 13, 15].

In the literature, SCS method proposed in [7, 16, 17] have better PAPR performance and low computational complexity compared to other methods such as Data Position Permutation (DPP) and conventional SLM. SCS manage to reduce PAPR by implementing shifting process on codeword without altering the structure of the original codeword itself. This opens up opportunities to further improve this method by manipulating the structure of the codeword before the shifting process take place. By doing so, more option can be made on how the bit position can be arrange in order to generate alternative codeword having a lower PAPR. The computational complexity of SCS method increases linearly as the number of shifting process increases which corresponds to the number of IFFT block used. Unfortunately, no research has been conducted to deal with SCS computational complexity issue without affecting its PAPR performance.

In this paper, a new technique to generate scramble data sequences based on SCS scheme called Median Codeword Shift (MCS) is proposed as a solution to the PAPR problem in OFDM system. MCS scheme is focusing on the development of alternative codeword by altering the structure of the original codeword before the shifting process is done between the serial to parallel and digital modulator. The advantages of the propose technique are the improvement of PAPR performance and lower computational complexity of the system as compared to SCS technique.

\section{PAPR IN OFDM SYSTEM}

Figure 1 illustrates the block diagram of OFDM system to be used in the following evaluation. To initiate the creation process of OFDM signal, the discrete binary stream need to be converted into an information symbol through a serial-to-parallel conversion process. Then, the information symbol will be modulated using 64-QAM modulator. The Inverse Fast Fourier Transform (IFFT) is implemented on the modulated symbol to transform it into an OFDM signal. OFDM signal is the sum of many independent signals modulated onto subchannels of equal bandwidth. For OFDM system having $N$ number of subcarriers, the input data block of length $N$ can be represented as $X=\left[X_{0}, X_{1}, \ldots, X_{N-1}\right]^{T}$ where $T$ is the symbol duration. Mathematically, the transmitting OFDM signal in complex time domain can be expressed as

$$
y(t)=\frac{1}{\sqrt{N}} \sum_{k=0}^{N-1} Y_{k} \cdot e^{j 2 \Pi f_{k} t}, \quad 0<t<N T
$$

Theoretically, PAPR is defined as the ratio between the peak power and its average power

$$
P A P R=10 \log \left(\frac{P_{\text {peak }}}{P_{\text {avg }}}\right) d B
$$

Therefore, PAPR in OFDM is given by

$$
P A P R=10 \log \left(\frac{\max |y(t)|^{2}}{E|y(t)|^{2}}\right) d B
$$


where $E|y(t)|^{2}$ indicates the average power. The Complementary cumulative distribution function (CCDF) is parameter is used to evaluate the performance of PAPR by estimating the probability of PAPR symbol that exceeds the threshold level of $P A P R_{0}$.

$$
C C D F\left(P A P R_{0}\right)=P_{r}\left(P A P R>P A P R_{0}\right)
$$

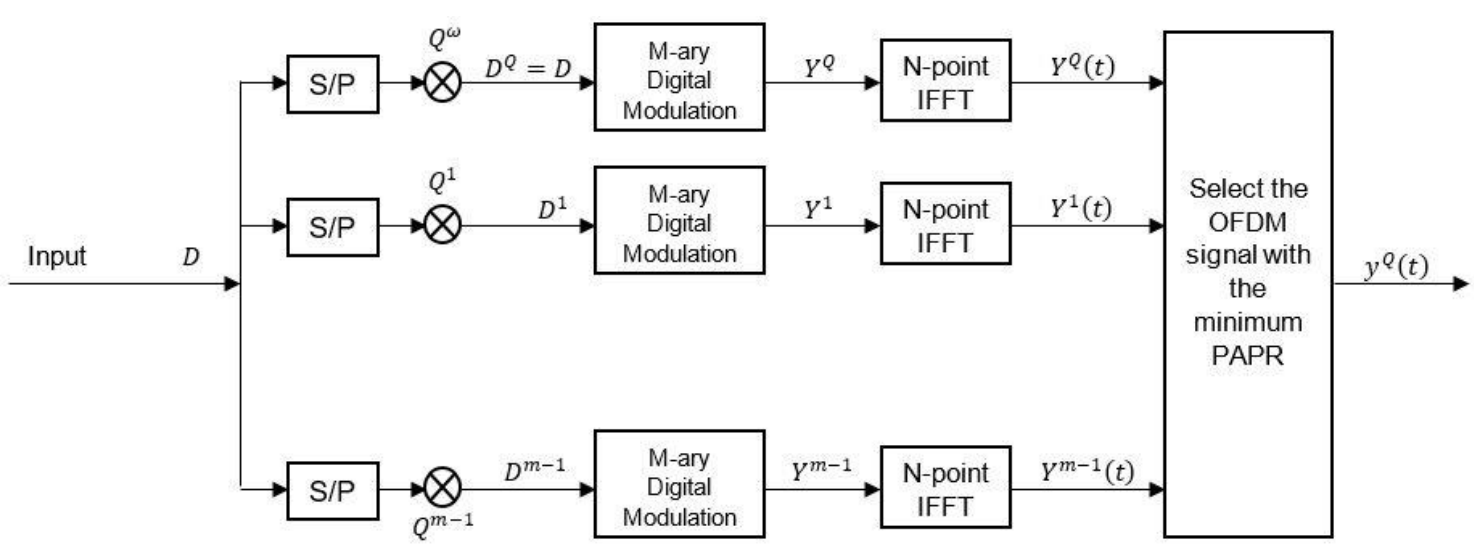

Figure 1. Block diagram of MCS technique at the transmitter in OFDM system

\section{PROPOSED MEDIAN CODEWORD SHIFT}

Data symbol $Y_{k}$ is one of the element that plays a significant role to determine the PAPR. Basically, data symbol $Y_{k}$ represents a sequence of sinusoidal waveform of duration $T$ and can be interpreted as:

$$
Y_{k}=D \cdot \phi_{j}(t)
$$

where $D$ indicates the codeword while $\phi_{j}(t)$ is the M-ary QAM digital modulation. In this paper, a technique to generate a scramble data sequence using permutation of data (circulant shift) in frequency domain is proposed. The working principle of this technique is to manipulate the structure of the codeword and the bit position in order to produce a new alternative sequence. This new sequence differ from the original sequence because the arrangement of the codeword is altered.

As shown in Figure 2, binary sequence codeword, $D$ is indicated as $D=\left[D_{1}, D_{2}, \ldots, D_{d}\right]$ where $d$ is the total number of input bits. After passing through the serial to parallel converter, these bits will be divided into several sub-block which can be denoted as $D=\left[D_{1}, D_{2}, \ldots, D_{K}\right]$ where $K=d / m$ and $m$ is representing the number of sub-block and the number of bit per symbol respectively. The divided codeword in each subblock can be written in the following pattern $D_{1}=\left[D_{1}, \ldots, D_{m}\right], D_{2}=\left[D_{m+1}, \ldots, D_{2 m}\right]$ and so on until $D_{K}$.

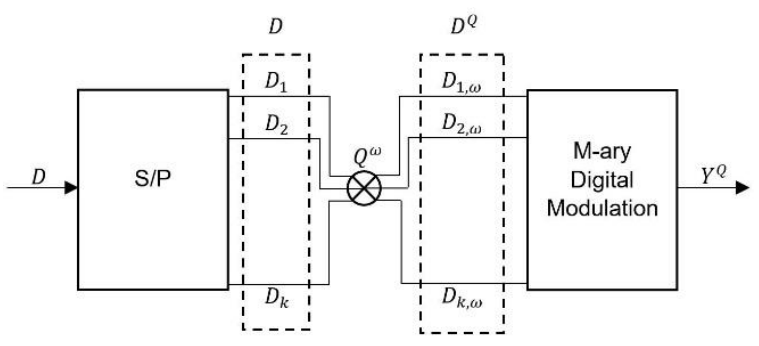

Figure 2. MCS sub-blocks

Et al shows the reconstruction of the codeword's structure by dividing it into two parts, A and B. The alternative codeword, $D^{Q}$ can be generated by circular rotation (one shift to the right) of the codeword for part A while part B remains in the idle state. After the shifting process in part A is completed, the process will be repeated for part B and this time around, part A will be in idle state. This process was applied to every sub-block of the codeword in Figure 1. 


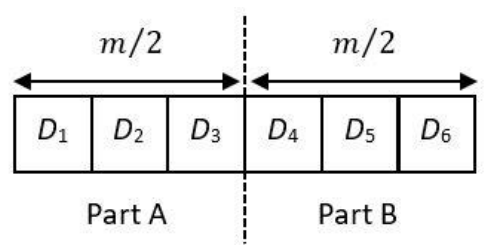

Et al. Structure of codeword for MCS method using 64-QAM

The total number of shifting, $\omega$ for both part A and part B can be expressed as:

$$
\omega=(m / 2)-1,0 \leq \omega \leq(m / 2)-1
$$

Table 1 summarizes the position of bits after the shifting process is performed. Codeword $D_{1,0}$ represents the initial position of the codeword bits. Codeword $D_{1,1}$ and $D_{1,2}$ represent the bits positon after the shifting process of part A while $D_{1,3}$ and $D_{1,4}$ for part B.

Table 1. Bit arrangement of codeword for MCS method using 64-QAM

\begin{tabular}{cc}
\hline Sub-block Codeword bits, $D_{k, \omega}$ & Position of Shifted Bits \\
\hline Codeword, $D_{1,0}$ & $D_{1}, D_{2}, D_{3}, D_{4}, D_{5}, D_{6}$ \\
Codeword Shift $1, D_{1,1}$ & $D_{3}, D_{1}, D_{2}, D_{4}, D_{5}, D_{6}$ \\
Codeword Shift $2, D_{1,2}$ & $D_{2}, D_{3}, D_{1}, D_{4}, D_{5}, D_{6}$ \\
Codeword Shift 3, $D_{1,3}$ & $D_{1}, D_{2}, D_{3}, D_{6}, D_{4}, D_{5}$ \\
Codeword Shift 4, $D_{1,4}$ & $D_{1}, D_{2}, D_{3}, D_{5}, D_{6}, D_{4}$ \\
\hline
\end{tabular}

The shifted codeword sequences can be denoted as $D^{Q}=\left[D_{1} \otimes Q^{\omega}, D_{2} \otimes Q^{\omega}, \ldots, D_{K} \otimes Q^{\omega}\right]$ and can be simplified as $D^{Q}=\left[D_{1, \omega}, D_{2, \omega}, \ldots, D_{k, \omega}\right]$ where $1 \leq Q \leq m$. Therefore, the new alternative OFDM symbol sequences can be written as:

$$
Y^{Q}=\prod_{k=1}^{K} D_{k, \omega} \cdot \phi_{j}(t)
$$

Hence, the number of transmitted OFDM signal in the time domain are given as below:

$$
y^{Q}(t)=\frac{1}{\sqrt{N}} \sum_{k=0}^{N-1} Y_{k}^{Q} \cdot e^{j 2 \Pi f_{k} t}
$$

Finally, the OFDM signal with lowest PAPR value will be selected to be transmitted. MCS technique requires side information to be transmitted along with the selected $y^{Q}$ signal for the purpose of data recovery at the receiver side.

\section{RESULTS AND DISCUSSION}

Simulation was carried out in order to evaluate and verify the efficacy of MCS. The simulation results obtained in this research are based on the 3rd Generation Partnership Project Long Term Evolution (3GPP-LTE) standards [18]. The simulation parameters are summarized in Table 2. To plot the CCDF, $10^{4}$ random OFDM symbols are taken into account. 
Table 2. Simulation parameters

\begin{tabular}{cc}
\hline Parameter & Value \\
\hline Bandwidth & $1.25 \mathrm{MHz}$ \\
Sampling frequency & $1.92 \mathrm{MHz}$ \\
Sampling time & $5.208 \times 10^{-7}$ second \\
IFFT size & 128 \\
Used subcarrier & 76 \\
Modulation & $64-\mathrm{QAM}$ \\
Guard interval & $1 / 4$ \\
Channel model & AWGN \\
\hline
\end{tabular}

The PAPR performance of conventional OFDM (denoted by original), SCS and MCS are shown in Figure 4. It is apparent that MCS managed to surpass the original signal and SCS. It is observed that MCS has improved the PAPR performance of up to $24 \%$ at $7.9 \mathrm{~dB}$ followed by the SCS which is $19.2 \%$ improvement at $8.4 \mathrm{~dB}$.

$Y_{k}^{Q}$ (eq.8) plays a significant role in MCS since this method deals with alternative codeword to reduce the PAPR value. In order to generate alternative codeword, MCS needs to execute two major processes, the reconstruction of the codeword structure and circularly shift process. By altering the structure of the codeword, MCS managed to reduce the codeword distance by half $(d=1, d=2)$ in comparison to the SCS which is at a distance of $(d=5)$. This is in line with the work in [19], that is reducing the distance of the codeword can improve the performance of PAPR.

The purpose of the shifting process in MCS is to generate alternative codeword so that the OFDM system can choose the OFDM symbol with the lowest power to be transmitted. In the conventional OFDM system, the system do not have the privilege to choose as it only generates one output. Therefore, by using MCS, the system can produce multiple output which can increases the probabilities of the system to obtain minimum PAPR with $24 \%$ improvement.

Figure 5 shows that PAPR value of SCS with 5 shifting process and having 6 IFFT block is much higher in comparison to the MCS with 4 shifting process and having 5 IFFT block. There was a $0.6 \mathrm{~dB}$ gap when both (SCS and MCS) were using the same number of IFFT block (IFFT block $=5$ ). The results of this study indicated that MCS has reduced the use of IFFT blocks by $16.67 \%$. These results reflect those finding in $[20,21]$ who also found that reduction in the number IFFT blocks will lead to reduction in computational complexity.

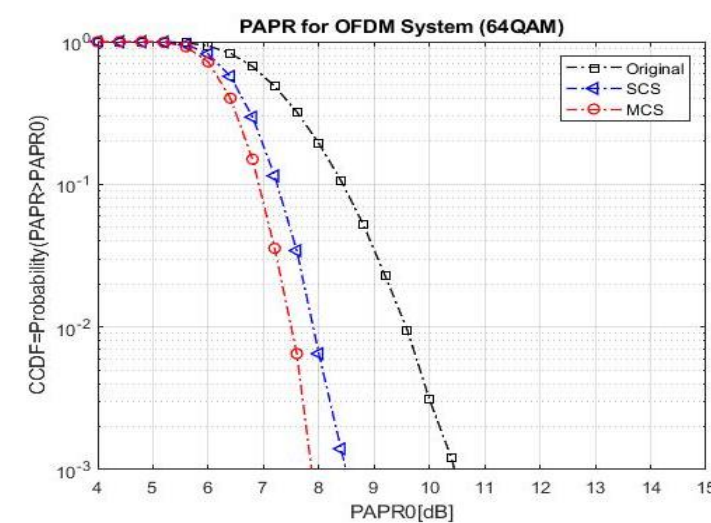

Figure 4. Comparison of PAPR reduction performance

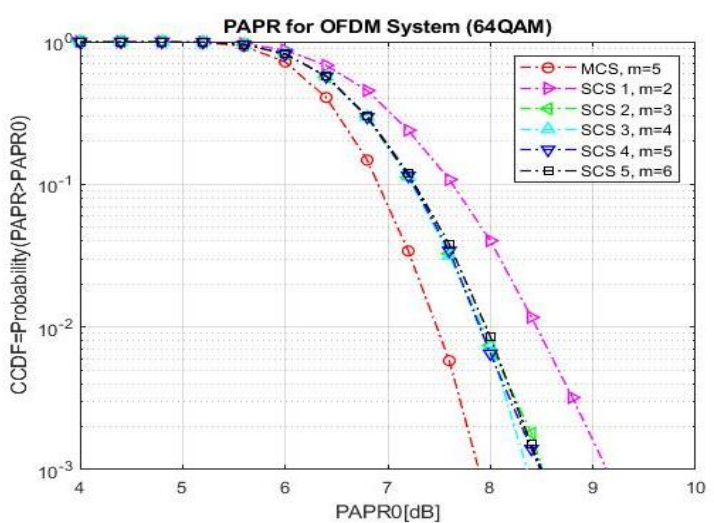

Figure 5. PAPR performance comparison for different number of IFFT blocks

\section{CONCLUSION}

In this paper, a new and efficient PAPR reduction method called MCS is proposed to solve the PAPR problem in OFDM system. The results have proven that MCS can achieved the objective with 24\% improvement in minimizing the PAPR as compared to the original OFDM system. In addition, MCS managed to beat SCS in term of PAPR reduction performance with $4.8 \%$ improvement. Furthermore, MCS can also reduce the computational complexity by reducing the usage of IFFT block in the system. Further research needs to be carry out to evaluate the PAPR performance of MCS scheme in different modulation techniques for other applications. 


\section{ACKNOWLEDGEMENTS}

We are grateful to the Faculty of Electrical Engineering, Universiti Teknologi Mara (UiTM) for providing the insight and expertise that greatly assist this research.

\section{REFERENCES}

[1] S. H. Han and J. H. Lee, "An Overview of Peak-to-Average Power Ratio Reduction Techniques for Multicarrier Transmission," IEEE Wireless Communications, vol. 12, pp. 56-65, 2005.

[2] A. Kumar and M. Gupta, "A Review on OFDM and PAPR Reduction Techniques," American Journal of Engineering and Applied Sciences, vol. 8, pp. 202-209, 2015.

[3] T. Jiang and Y. Wu, "An Overview: Peak-to-Average Power Ratio Reduction Techniques for OFDM Signals," IEEE Transactions on Broadcasting, vol. 54, pp. 257-268, 2008.

[4] S. P. Yadav and S. C. Bera, "PAPR Reduction for Improved Efficiency of OFDM Modulation for Next Generation Communication Systems," International Journal of Electrical and Computer Engineering (IJECE), vol. 6, pp. 2310-2321, 2016

[5] H. A. Saadi, et al., "A PAPR Reduction for OFDM Signals Based on Self-Adaptive Multipopulation DE algorithm," International Journal of Electrical and Computer Engineering (IJECE), vol. 7, pp. 2365-2373, 2017.

[6] M. Sabbaghian, et al., "Near Shannon Limit and Low Peak To Average Power Ratio Turbo Block Coded OFDM," IEEE Transactions on Communications, vol. 59, pp. 2042-2045, 2011.

[7] E. Abdullah, et al., "Minimizing High PAPR in OFDM System Using Circulant Shift Codeword," Jurnal Teknologi, vol. 78, pp. 135-140, 2016.

[8] C. Nie and Y. Bai, "PAPR Reduction with Amplitude Clipping and Subband Filter in Filtered-OFDM System," First International Conference on 5G for Future Wireless Networks, 5GWN 2017, pp. 220-227, 2017.

[9] J. A. Davis and J. Jedwab, "Peak-to-Mean Power Control in OFDM, Golay Complementary Sequences, and ReedMuller codes," IEEE Transactions on Information Theory, vol. 45, pp. 2397-2417, 1999.

[10] S. H. Muller and J. B. Huber, "OFDM with Reduced Peak-to-Average Power Ratio by Optimum Combination of Partial Transmit Sequences,” Electronics Letters, vol. 33, pp. 368-369, 1997.

[11] K. C. Chung, et al., "Low Complexity PTS Algorithms with Error Correction Capability in OFDM Systems," 2015 Seventh International Conference on Ubiquitous and Future Networks, pp. 254-256, 2015.

[12] R. W. Bauml, et al., "Reducing the Peak-to-Average Power Ratio of Multicarrier Modulation by Selected Mapping," Electronics Letters, vol. 32, pp. 2056-2057, 1996.

[13] S. Y. Zhang and B. Shahrrava, "A Selected Mapping Technique Using Interleavers for PAPR Reduction in OFDM Systems," Wireless Personal Communications, vol. 99, pp. 329-338, 2018.

[14] P. Manhas and M. K. Soni, "OFDM PAPR Reduction Using Recurring SLM with Cyclic and Linear Block Codes Schemes," TELKOMNIKA Indonesian Journal of Electrical Engineering, vol. 16, pp. 1-6, 2015.

[15] P. Boonsrimuang, et al., "Proposal of New PAPR Reduction Method for OFDM Signal by Using Permutation Sequences," Advanced Communication Technology (ICACT), 2012 14th International Conference, vol. 2, pp. 342$345,2012$.

[16] E. Abdullah, et al., "PAPR Reduction Using SCS-SLM Technique in STFBC MIMO-OFDM," ARPN Journal of Engineering and Applied Sciences, vol. 12, pp. 3218-3221, 2017.

[17] E. Abdullah, et al., "Modified Selective Mapping Scheme with Low Complexity for Minimizing High PeakAverage Power Ratio in Orthogonal Frequency Division Multiplexing System," AIP Conference Proceedings, vol. 1774, 2016.

[18] T. D. Chiueh and P. Y. Tsai, "OFDM Baseband Receiver Design for Wireless Communications," Singapore, John Wiley \& Sons (Asia) Pte Ltd, 2007.

[19] K. G. Paterson and V. Tarokh, "On the Existence and Construction of Good Codes with Low Peak-to-Average Power Ratios," IEEE Transactions on Information Theory, vol. 46, pp. 1974-1987, 2000.

[20] S. J. Heo, et al., "A Modified SLM Scheme with Low Complexity for PAPR Reduction of OFDM Systems," IEEE Transactions on Broadcasting, vol. 53, pp. 804-808, 2007.

[21] D. W. Lim, et al., "A New SLM OFDM Scheme with Low Complexity for PAPR Reduction," IEEE Signal Processing Letters, vol. 12, pp. 93-96, 2005.

\section{BIOGRAPHIES OF AUTHORS}

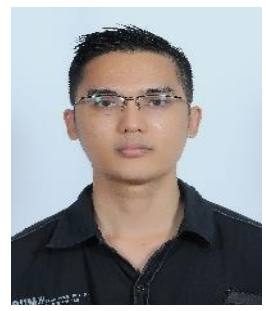

Mohd Danial Rozaini was born in Kuala Lumpur, Malaysia. He received a Bachelor of Engineering (Hons) Electronics Engineering (Communication) from Universiti Teknologi MARA (UiTM), Malaysia in 2014. He received a Master of Science in Telecommunication and Information Engineering from Universiti Teknologi MARA (UiTM) in 2016. He is currently working towards his $\mathrm{PhD}$ at the Faculty of Electrical Engineering, Universiti Teknologi MARA (UiTM). His research interests mainly focus on wireless communications, OFDM systems and power consumption reduction in wireless communications devices. 

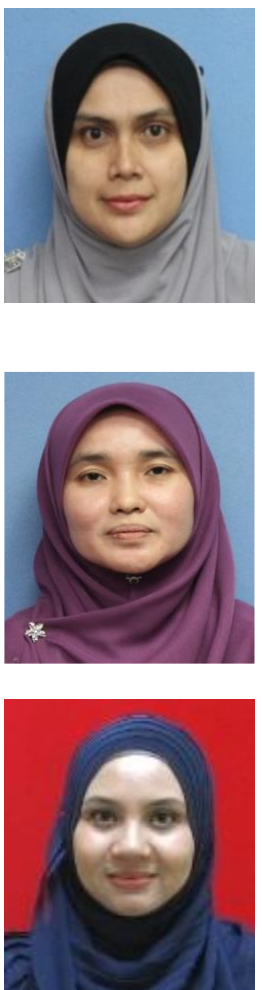

Azlina Idris is a Senior Lecturer at the Universiti Teknologi MARA (UiTM), Selangor, Malaysia. She obtained her $\mathrm{PhD}$ in wireless communication from University Malaya (UM), Malaysia. She has received the Master of Engineering in Electrical from Universiti Teknologi Malaysia (UTM). Previously, she obtained her first degree from Leeds Metropolitan University, United Kingdom with Honours, in Applied Computer. She is a member of Wireless Communication Technology (WiCOT) Research Interest Group (RIG) and her research interests include OFDM/OFDMA transmission, single and multiuser precoding, modulation, MIMO transmission techniques and receivers, channel coding, interference management and mitigation, and channel modeling (channel estimation).

Darmawaty Mohd Ali is a Senior Lecturer at the Universiti Teknologi MARA (UiTM), Selangor, Malaysia. She obtained her PhD in 2012 from Universiti Malaya (UM), Malaysia. She has received the Master of Engineering in Electrical in 2002 from Universiti Teknologi Malaysia (UTM). Previously, she obtained her first degree from Universiti Kebangsaan Malaysia (UKM) with Honours, in Electrical, Electronic and System, graduating in 1999. She is a member of Wireless Communication Technology (WiCOT) Research Interest Group (RIG) and her research interests include Wireless Access Technology and Quality of Service in Wireless Broadband.

Ezmin Abdullah is currently working as a senior lecturer in Centre for Computer Engineering Studies, Faculty of Electrical Engineering, Universiti Teknologi MARA (UiTM), Selangor, Malaysia. She was graduated from Hirosaki University, Japan in 2006 for her bachelor degree, major in Electronics and Information Systems Engineering. She received her masters and PhD degree from Universiti Teknologi MARA in 2013 and 2017 respectively. Her research interests are wireless communications, OFDM systems, power consumption reduction in wireless communications devices, FPGA applications and energy management. 